\title{
Clobetasol propionate enhances neural stem cell and oligodendrocyte differentiation
}

\author{
WENTAO SHI $^{1 *}, \mathrm{SHIQI} \mathrm{BI}^{2 *}, \mathrm{YAO} \mathrm{DAI}^{2 *}, \mathrm{KAIYUAN} \mathrm{YANG}^{2}$, YOUFA ZHAO $^{1}$ and ZHIJIAN ZHANG ${ }^{2}$ \\ ${ }^{1}$ Department of Orthopedics, Gaochun People's Hospital, Nanjing, Jiangsu 211300; \\ ${ }^{2}$ Department of Embryology, School of Medicine, Jiangsu University, Zhenjiang, Jiangsu 212001, P.R. China
}

Received June 21, 2018; Accepted May 16, 2019

DOI: $10.3892 /$ etm.2019.7692

\begin{abstract}
Clobetasol propionate (Clo) is a potent topical glucocorticoid and a potential remyelinating agent that has been approved by the U.S. Food and Drug Administration. However, the effect of Clo on neural stem cells (NSCs) remains largely unknown. The aim of the present study was to investigate the effect of Clo on the differentiation of NSCs in vitro. NSCs were isolated from mouse embryonic brain tissues and expanded in vitro. The effect of Clo on NSC viability was examined using an MTT assay. Differentiating NSCs were treated with 5 or $10 \mu \mathrm{M}$ Clo, or with DMSO control, and the degree of differentiation was examined following culture in stem cell differentiation induction medium for 7 days. The effect of Clo on NSC differentiation was assessed using immunocytochemistry and western blot analyses. The results revealed that Clo significantly increased NSC viability compared with the DMSO control group. Treatment with Clo also significantly increased the number of NSCs that differentiated into growth associated protein 43 positive neurons and corresponding axon lengths were also significantly increased. In addition, treatment with Clo significantly increased the number of myelin basic protein positive oligodendrocytes and decreased the number of glial fibrillary acidic protein positive astrocytes. Furthermore, inhibition of the sonic hedgehog and AMP-activated protein kinase signaling pathways inhibited Clo-induced NSC differentiation, and treatment with Clo upregulated the expression of several neurotrophic factors. In conclusion, the results of the current study suggest that Clo may have a potential therapeutic
\end{abstract}

Correspondence to: Dr Zhijian Zhang, Department of Embryology, School of Medicine, Jiangsu University, 301 Xuefu Road, Zhenjiang, Jiangsu 212001, P.R. China

E-mail: zzj@ujs.edu.cn

Dr Youfa Zhao, Department of Orthopedics, Gaochun People's Hospital, 9 Chunzhong Road, Nanjing, Jiangsu 211300, P.R. China E-mail: zhaoyf1955@163.com

*Contributed equally

Key words: neural stem cells, clobetasol propionate, sonic hedgehog, differentiation benefit in neurological disorders affecting oligodendrocytes and neurons.

\section{Introduction}

The central nervous system (CNS) does not spontaneously regenerate following injury, a theory that has been widely accepted since it was initially devised in the early 20th century, by the Spanish neuroanatomist Santiago Ramon y Cajal (1). The lack of axonal regeneration in the adult mammalian CNS, particularly in the spinal cord, is due to a combination of factors, including demyelination and injury-induced glial scars (2). Following spinal cord injury, the microenvironment of the spinal cord and brain contains endogenous neural stem cells (NSCs); however, there is no evidence of de novo neurogenesis upon injury (3). Numerous studies have demonstrated that the fate of NSCs is regulated locally by the niche microenvironment and that this is influenced by a number of factors, including specific hormones, such as dexamethasone, growth hormone and prolactin $(4,5)$. Numerous compounds and hormones have been identified to affect NSC differentiation and proliferation $(6,7)$, which suggests that regeneration of the injured spinal cord may be possible.

NSCs are multipotent cells capable of self-renewal and differentiation into multiple cell types, including neurons, astrocytes and oligodendrocytes (8). As such, they are a powerful research tool for examining the mechanisms underlying CNS diseases, as well as therapeutic strategies. Mature NSCs contribute to new memory formation and mediate the response to neural injury $(9,10)$. Therefore, NSC proliferation and differentiation serve an important role in neural development, maintenance and regeneration. However, there is a tendency for NSCs to differentiate into astrocytes, which may form glial scars that suppress spontaneous remyelination and neural recovery (11). Therefore, a compound that promotes endogenous NSC proliferation and differentiation is likely to also promote neuronal repair in the CNS.

Clobetasol propionate (Clo), which has been approved by the U.S. Food and Drug Administration, is a corticosteroid and a member of the glucocorticoid family that is widely used to treat a number of skin disorders, including herpes labialis, psoriasis and lichen sclerosus (12). Clo is also a potential remyelinating agent that has been demonstrated to promote differentiation in oligodendrocyte precursor cell (OPC) 
cultures and remyelination in vivo (12-15). Clo enhances the differentiation of ectodermal stem cell-derived OPCs into oligodendrocytes, which has been validated in vivo $(16,17)$.

Clo promotes the proliferation of primary neuronal precursor cells alone and synergistically in the presence of sonic hedgehog (SHH) protein (18). Therefore, Clo may function as an important modulator for the proliferation and differentiation of NSCs; however, to the best of the authors' knowledge, no study to date has examined the direct effect of Clo on cultured NSCs.

The aim of the present study was to determine the effect of Clo on the differentiation of NSCs into neurons, oligodendrocytes and astrocytes in vitro. The results of the current study demonstrate that Clo has potential as a disease-modifying therapy that may have applications in neurological disorders that affect neurons and oligodendrocytes.

\section{Materials and methods}

Cell culture. Pregnant (E14) adult Sprague-Dawley (SD) rats ( $\mathrm{n}=5$; weight, $250 \mathrm{~g}$; age, 12 weeks; supplied by the Animal Center of Jiangsu University) were used in the present study. The present study, including all animal experimental protocols, was approved by the Jiangsu University Animal Care Committee (Jiangsu, China; protocol number 2016-08-07). Pregnant (E14) SD rats were sacrificed by the intraperitoneal injection of pentobarbital $(200 \mathrm{mg} / \mathrm{kg})$. Embryonic rats were dissected from the uterine horn under sterile conditions and placed in PBS. The embryonic brains were immediately harvested by microdissection, and maintained in PBS prior to dissociation. Briefly, cells were dissociated by mechanical shearing followed by filtration through a $40-\mu \mathrm{m}$ mesh. Cell suspensions were prepared, and primary embryonic neurospheres cultured as previously described (19). The cells were seeded in ultra-low-attachment culture plates (Corning Inc.) and cultured in serum-free DMEM/F12 (Gibco; Thermo Fisher Scientific, Inc) supplemented with $2 \mathrm{mM}$ L-glutamine (Invitrogen; Thermo Fisher Scientific, Inc.), $20 \mathrm{ng} / \mathrm{ml}$ basic fibroblast growth factor, $20 \mathrm{ng} / \mathrm{ml}$ epidermal growth factor (both PeproTech, Inc.), 2\% B27 (Gibco; Thermo Fisher Scientific, Inc.) and $100 \mathrm{U} / \mathrm{ml}$ penicillin/streptomycin (Beijing Solarbio Science \& Technology Co., Ltd.). All cultures were maintained at $37^{\circ} \mathrm{C}$ in a humidified atmosphere of $5 \% \mathrm{CO}_{2}$. After passage 3 , NSCs were identified by their morphological characteristics and nestin expression, as described below. The subcultured neurospheres were characterized by immunofluorescence staining with anti-nestin antibody. Neurospheres formed from NSCs of passage three were used for subsequent experiments.

Cell viability assay. The cell viability of NSCs was assessed using the MTT colorimetric assay (Beijing Solarbio Science \& Technology Co., Ltd.). Briefly, dissociated neurosphere-derived cells from primary cultures were seeded onto non-treated 96-well plates (Corning Inc.) at a density of $5 \times 10^{3}$ cells/well and grown for $48 \mathrm{~h}$ following treatment with different concentrations $(2.5,5,7.5,10,12.5$ and $15 \mu \mathrm{M})$ of Clo (Selleck Chemicals), dissolved in dimethyl sulfoxide (DMSO; Sigma-Aldrich; Merck KGaA) to a final concentration of $0.01 \%$, or DMSO alone as a control. After the 48-h treatment, the Clo-containing medium was removed, the cells were gently washed twice with PBS and then $200 \mu \mathrm{l}$ MTT $(0.5 \mathrm{mg} / \mathrm{ml})$ in PBS was added to each well. Following incubation with MTT for $4 \mathrm{~h}$ at $37^{\circ} \mathrm{C}$, the formazan dye in DMSO produced by viable cells was quantified by measurement of the absorbance at $460 \mathrm{~nm}$ using a microplate reader. Five independent experiments were conducted. In each experiment, 15 replicates were measured for each concentration.

Neurosphere counting. Separated neurosphere-derived cells were seeded onto ultra-low-attachment 96-well culture plates at a density of $5 \times 10^{3}$ cells/well. Cells were treated with different concentrations $(2.5,5,7.5,10,12.5$ and $15 \mu \mathrm{M})$ of Clo dissolved in DMSO or with DMSO alone as a control (each treatment condition repeated in 15 wells). Cells were cultured in serum-free DMEM/F12 (Gibco; Thermo Fisher Scientific, Inc) supplemented with $2 \mathrm{mM}$ L-glutamine (Invitrogen; Thermo Fisher Scientific, Inc.), $20 \mathrm{ng} / \mathrm{ml}$ basic fibroblast growth factor, $20 \mathrm{ng} / \mathrm{ml}$ epidermal growth factor (both PeproTech, Inc.), 2\% B27 (Gibco; Thermo Fisher Scientific, Inc.) for 5 days. The total number of neurospheres with a diameter $>50 \mu \mathrm{m}$ was counted in each well using an inverted light microscope (Zeiss Axio Observer; Zeiss GmbH).

NSC differentiation. In all experiments NSCs were cultured in serum-free DMEM/F12 for $12 \mathrm{~h}$, unless otherwise stated. For NSC differentiation assays, $100-\mu \mathrm{m}$-diameter neurospheres were collected by centrifugation $\left(1,000 \mathrm{x}\right.$ g for $10 \mathrm{~min}$ at $\left.4^{\circ} \mathrm{C}\right)$ and 10-15 neurospheres were seeded onto a 48 -well plate (BD Biosciences) in stem cell differentiation induction medium [DMEM/F12 containing 4\% fetal bovine serum (Gibco; Thermo Fisher Scientific, Inc.), 2\% B27 and 2\% N2]. Following 12-h culture, differentiating NSCs were treated with Clo (5 or $10 \mu \mathrm{M}$ ) or DMSO (control) at $37^{\circ} \mathrm{C}$ and differentiation was evaluated after culture in stem cell differentiation induction medium after 7 days of treatment.

SHH inhibition. To establish whether the SHH signaling pathway is involved in NSC differentiation, the SHH signaling pathway inhibitor cyclopamine (CYC; Sigma-Aldrich; Merck $\mathrm{KGaA}$ ) was used to treat NSCs. Prior to inhibition experiments, NSCs were cultured in stem cell differentiation induction medium and treated with Clo $(10 \mu \mathrm{M})$ for 7 days, as described above. Cells were treated with $10 \mu \mathrm{M} \mathrm{CYC}$ diluted in stem cell differentiation induction medium for 7 days.

5'-AMP-activated protein kinase (AMPK) inhibition. To establish whether the AMPK signaling pathway is involved in NSC differentiation, the AMPK signaling pathway inhibitor Compound C (CC; Sigma-Aldrich; Merck KGaA) was used to treat NSCs. NSCs were cultured in stem cell differentiation induction medium and treated with Clo $(10 \mu \mathrm{M})$ for 7 days, as described above. Cells were treated with $5 \mu \mathrm{M} \mathrm{CC}$ diluted in stem cell differentiation induction medium for 7 days.

Immunocytochemistry and western blot analysis. Immunocytochemistry was used to determine the expression pattern of differentiated cell markers in NSCs. Cells were washed three times with PBS and fixed with $4 \%$ paraformaldehyde at $4^{\circ} \mathrm{C}$ for $12 \mathrm{~h}$. Subsequently, cells were blocked with 

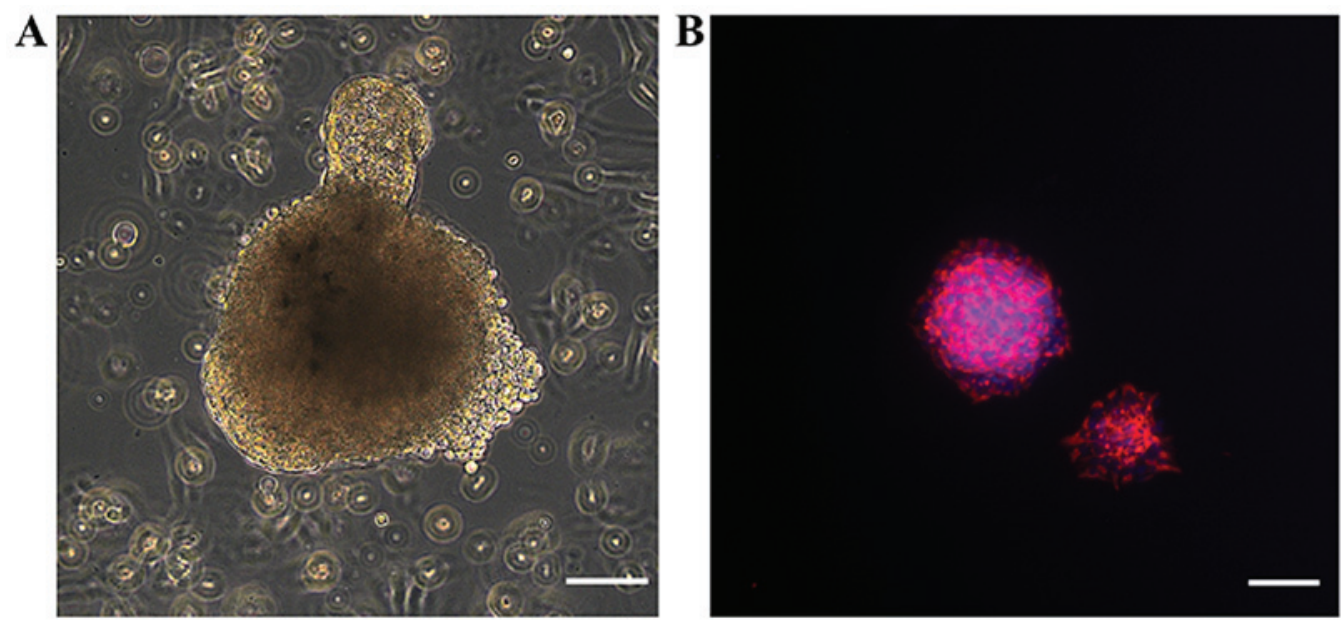

Figure 1. Characterization of NSCs. (A) Morphology of neurospheres cultured for 7 days in serum-free medium. (B) Immunostaining of NSCs with nestin antibody and DAPI. The majority of cells in the neurospheres were positive for the NSC marker nestin. Scale bar= $25 \mu \mathrm{m}$. NSCs, neural stem cells.

$0.1 \%$ Triton X-100 in PBS supplemented with $3 \%$ bovine serum albumin (Beijing Solarbio Science \& Technology Co., Ltd.) at $37^{\circ} \mathrm{C}$ for $45 \mathrm{~min}$. Cells were incubated with the following primary antibodies: Anti-growth associated protein 43 (GAP-43; rabbit polyclonal; 1:200; cat. no. PA-1037), anti-myelin basic protein (MBP; rabbit polyclonal; 1:200; cat. no. BA0094), anti-nestin (1:200; cat. no. PB0920), anti-glial fibrillary acidic protein (GFAP; mouse monoclonal; 1:200; cat. no. PB0046; all Boster Biological Technology), anti-SHH (1:200; rabbit monoclonal; cat. no. ab53281; Abcam), anti-phosphorylated adenosine 5'-monophosphate (p-AMP)-activated protein kinase (p-AMPK; rabbit polyclonal; 1:200; cat. no. sc-25792) and anti-phosphorylated mitogen-activated protein kinase (p-ERK; rabbit polyclonal; 1:200; cat. no. sc-514302; both Santa Cruz Biotechnology, Inc.) for $12 \mathrm{~h}$ at $4^{\circ} \mathrm{C}$. Following primary antibody incubation, cells were washed three times with PBS and then incubated with secondary antibodies, Cy3-labeled goat anti-mouse/rabbit IgG (1:200; cat. no. C5838/C2821 Sigma-Aldrich; Merck KGaA) and Alexa Fluor 488-conjugated goat anti-mouse IgG (1:200; cat. no. SAB4600388; Sigma-Aldrich; Merck KGaA) for $3 \mathrm{~h}$ at room temperature. The nuclei were counterstained with DAPI (Sigma-Aldrich) for $30 \mathrm{~min}$ at room temperature and images were acquired using a fluorescent microscope (Zeiss Axio Observer; Zeiss GmbH).

For western blot analysis, total protein from adherent cells in each experimental group was harvested. Total protein was extracted from cells using radioimmunoprecipitation assay buffer (Beijing Solarbio Science \& Technology Co., Ltd.) containing PMSF, phosphatase inhibitor cocktail and EDTA (all Beijing Solarbio Science \& Technology Co., Ltd.). Total protein was quantified using a Bradford protein assay and $15 \mu \mathrm{g}$ protein/lane was separated via SDS-PAGE on a $10 \%$ gel. The separated proteins were subsequently transferred onto PVDF membranes (EMD Millipore) and blocked for $1 \mathrm{~h}$ at room temperature with $3 \%$ non-fat dried milk powder in TBS at room temperature. The membranes were incubated with primary antibodies against: GAP-43 (rabbit polyclonal; 1:200; cat. no. PA-1037), GFAP (mouse monoclonal; 1:200; cat. no. PB0046), MBP (MBP; rabbit polyclonal; 1:200; cat. no. BA0094), brain derived neurotrophic factor (BDNF; rabbit polyclonal; 1:200; cat. no. PB0013), neurotrophins-3 (NT-3; mouse polyclonal; 1:200; cat. no. PB0886) and $\beta$-tubulin (1:200; cat. no. BM1453) all from Wuhan Boster Biological Technology, Ltd.; SHH (1:200; rabbit monoclonal; cat. no. ab53281) and SMO (1:200; rabbit monoclonal; cat. no. ab236465) both from Abcam; and p-AMPK/AMPK (rabbit polyclonal; 1:200; cat. nos. sc-25792 and sc-74461, respectively) and p-ERK/ERK (rabbit polyclonal; 1:200; cat. nos. sc-81492 and sc-514302, respectively) from Santa Cruz Biotechnology, Inc., overnight at $4{ }^{\circ} \mathrm{C}$. Following primary antibody incubation, membranes were incubated with HRP-conjugated secondary antibodies (1:200; cat. nos. BA1050 and BA1056; Wuhan Boster Biological Technology, Ltd.) for $1 \mathrm{~h}$ at room temperature. Protein bands were visualized using an ECL kit (EMD Millipore). Chemiluminescent signals were captured digitally using a chemiluminescence imaging system. The images were analyzed using ImageJ software (version $1.51 \mathrm{k}$; National Institutes of Health).

Statistical analysis. Data are presented as the mean \pm standard error of the mean. All statistical analyses were performed using SPSS software (version 22.0; IBM Corp.,). One-way analysis of variance followed by protected Fisher's least significant difference post hoc test was used to analyze differences among multiple groups. $\mathrm{P}<0.05$ were considered to indicate a statistically significant difference.

\section{Results}

Characterization of NSCs. Primary NSCs formed neurospheres of various sizes, with diameters of 50-100 $\mu \mathrm{m}$ after 7 days of culture in serum-free medium (Fig. 1A). To characterize NSCs, immunocytochemical staining of nestin, a commonly used NSC marker, was performed (Fig. 1B). Strong staining of nestin was observed in the cultured neurospheres.

Effect of Clo on NSC proliferation. To determine the effect of Clo on NSCs, cell viability was examined using the MTT assay following treatment with different concentrations (2.5, 

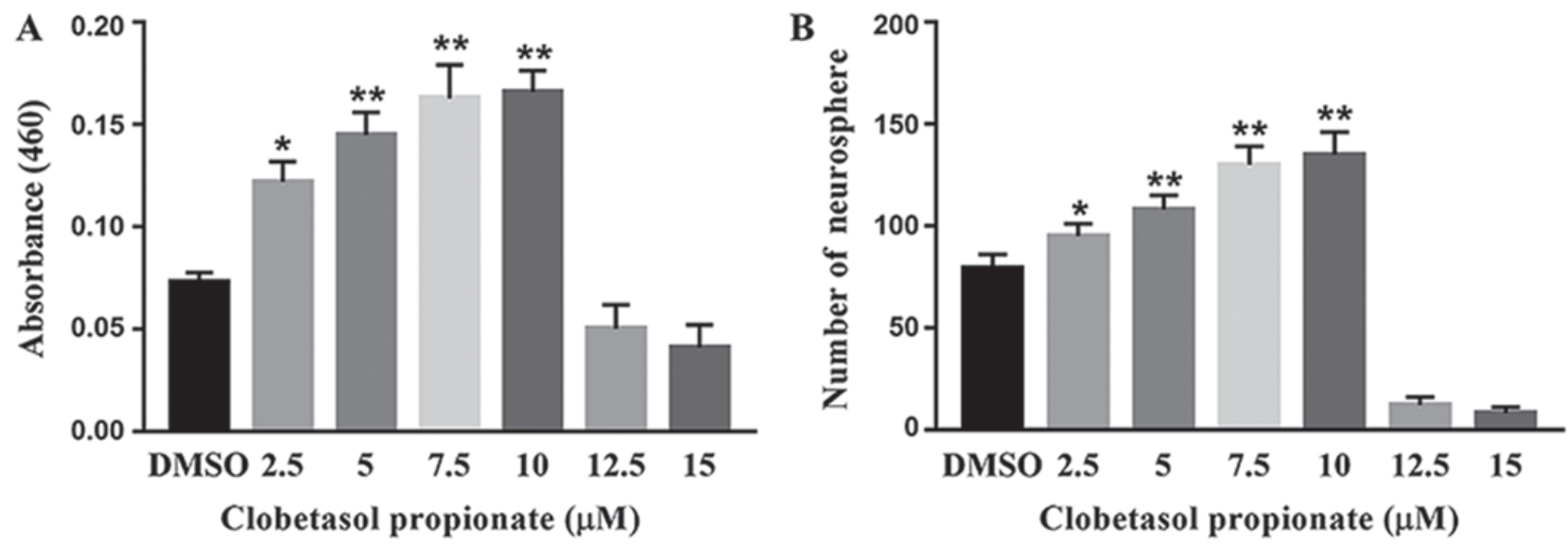

Figure 2. Effect of Clo on NSC proliferation. (A) MTT assay was used to examine NSC cell viability following treatment with different concentrations of Clo. (B) The number of neurospheres formed increased significantly following treatment with $\leq 10 \mu \mathrm{M}$ concentrations of Clo. Data are presented as the mean \pm standard error of the mean. $\mathrm{n}=15 .{ }^{*} \mathrm{P}<0.05$ and ${ }^{* * *} \mathrm{P}<0.01$ vs. DMSO. Clo, clobetasol propionate; NSC, neural stem cell.
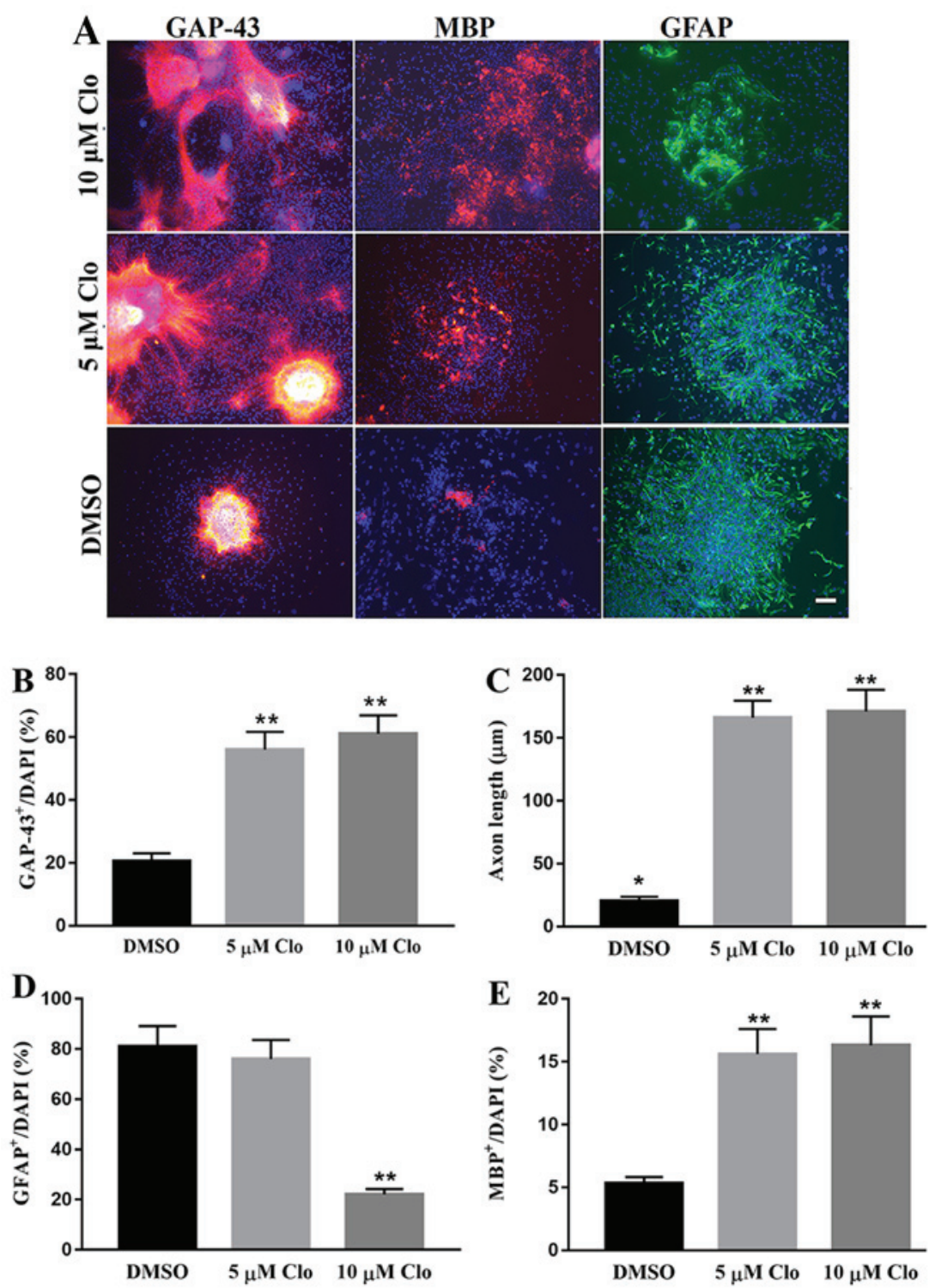

Figure 3. Effect of Clo on NSC differentiation. NSCs were cultured in specific differentiation induction medium. (A) Expression of GAP-43, GFAP and MBP in differentiating NSCs following treatment with Clo (5 or $10 \mu \mathrm{M}$ ) or DMSO (control) as detected by immunocytochemistry. Scale bar=25 $\mu \mathrm{m}$. (B) Proportion of GAP-43 positive cells in the NSCs. (C) Mean axon length in GAP-43 positive NSCs. Quantitative analysis of (D) GFAP positive and (E) MBP positive NSCs. $\mathrm{n}=5$. ** $\mathrm{P}<0.01$ vs. DMSO. Clo, clobetasol propionate; NSCs, neural stem cells; GAP-43, growth associated protein 43; GFAP, glial fibrillary acidic protein; MBP, myelin basic protein. 

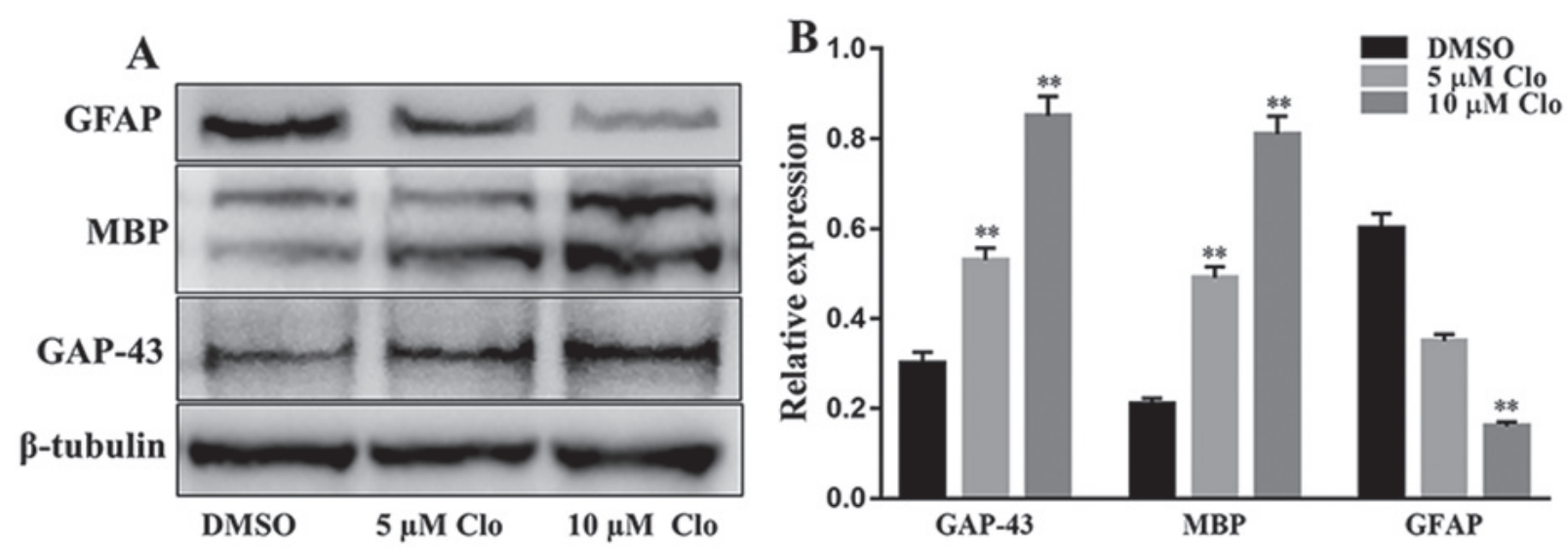

Figure 4. Effect of Clo on GAP-43, GFAP and MBP expression in differentiated NSCs. (A) Representative western blots of GAP-43, GFAP and MBP in differentiated NSCs following treatment with Clo $(5$ or $10 \mu \mathrm{M})$ or DMSO (control). (B) Quantification of GAP-43, GFAP and MBP protein expression. Data are presented as the mean \pm standard error of the mean. $n=5$. ${ }^{* *} \mathrm{P}<0.01$ vs. DMSO. Clo, clobetasol propionate; GAP- 43 , growth associated protein 43 ; GFAP, glial fibrillary acidic protein; MBP, myelin basic protein; NSCs, neural stem cells.

5, 7.5, 10,12.5 and $15 \mu \mathrm{M})$ of Clo. Treatment with Clo significantly increased NSC viability at lower concentrations $(2.5$, $5,7.5$ and $10 \mu \mathrm{M}$ ) compared with DMSO (Fig. 2A). However, cell death was observed following treatment with Clo at concentrations $\geq 12.5 \mu \mathrm{M}$. These data indicate that treatment with Clo increases NSC proliferation at low concentrations. Similarly, treatment with Clo significantly increased neurosphere formation at lower concentrations, with a peak number of neurospheres obtained with a Clo concentration of $10 \mu \mathrm{M}$ (Fig. 2B).

Effect of Clo on NSC differentiation. To study the effect of Clo on NSC differentiation, NSCs were cultured in specific differentiation induction medium with or without Clo (5 and $10 \mu \mathrm{M})$. NSC differentiation was confirmed by immunocytochemistry using neural cell lineage-specific antibodies, which included GAP-43 (neurons), GFAP (astrocytes) and MBP (oligodendrocytes; Fig. 3A). Treatment with Clo significantly increased the number of GAP-43 positive neurons compared with the DMSO control group (Fig. 3B). In addition, treatment with Clo significantly increased the axon length in GAP-43 positive neurons compared with the DMSO control group (Fig. 3C). No significant difference in axon length was observed between the 5 and $10 \mu \mathrm{M}$ groups (Fig. 3C). No significant difference was detected in astrocyte differentiation between the $5 \mu \mathrm{M}$ Clo group and the DMSO control group (Fig. 3D). However, treatment with a higher concentration $(10 \mu \mathrm{M})$ of Clo significantly decreased the number of differentiated GFAP positive astrocytes compared with that in the $5 \mu \mathrm{M}$ Clo or DMSO control group (Fig. 3D). Treatment with Clo significantly increased the number of MBP positive oligodendrocytes compared with that in the DMSO control group (Fig. 3E). No significant difference was observed between the 5 and $10 \mu \mathrm{M}$ groups (Fig. 3E).

Furthermore, the protein expression levels of GAP-43, MBP and GFAP were determined by western blot analysis in differentiated NSCs following treatment with Clo $(5$ or $10 \mu \mathrm{M})$ or DMSO (Fig. 4A). Treatment with Clo significantly increased GAP-43 and MBP protein expression levels, and significantly decreased GFAP protein expression levels compared with the corresponding levels in the DMSO control group (Fig. 4B).
Effect of Clo on p-AMPK protein expression. Given the role of ERK and AMPK signaling pathways in stem cell proliferation and differentiation $(20,21)$, the expression of SHH, p-AMPK and p-ERK proteins was examined in NSCs cultured in specific differentiation induction medium with or without Clo (5 and $10 \mu \mathrm{M})$ using immunocytochemistry (Fig. 5A) and western blotting (Fig. 5B and C). No significant difference in the level of p-ERK was observed between the Clo and DMSO treatment groups ( $\mathrm{P}>0.05$; Fig. 5B and $\mathrm{C})$. However, western blot analysis demonstrated that the level of p-AMPK was significantly increased in differentiating NSCs following treatment with Clo compared with the DMSO control group $(\mathrm{P}<0.05$; Fig. 5B and C).

Effect of Clo on SHH and SMO protein expression. The protein expression levels of SHH and SMO were determined in differentiated NSCs following treatment with Clo (5 or $10 \mu \mathrm{M}$ ) or DMSO (Fig. 5). Immunocytochemistry indicated that treatment with Clo markedly increased SHH protein expression levels (Fig. 5A), and western blotting confirmed that SHH and SMO were significantly increased in the Clo treatment groups compared with the DMSO control group (Fig. 5B and C). These results suggest that treatment with Clo affects SHH signaling and this may provide a mechanistic explanation for the enhanced differentiation of NSCs into neurons and oligodendrocytes in the presence of Clo.

Inhibition assays. To further examine the involvement of AMPK and SHH signaling pathways in NSC differentiation, the SHH pathway inhibitor CYC and the AMPK pathway inhibitor CC were used to block these pathways and NSC differentiation was examined. The protein expression levels of GAP-43, MBP, GFAP, SHH and SMO were determined by western blot analysis in differentiated NSCs following treatment with $10 \mu \mathrm{M}$ Clo and $10 \mu \mathrm{M} \mathrm{CYC} \mathrm{(Fig.} \mathrm{6A).} \mathrm{Treatment}$ with Clo alone significantly increased GAP-43, MBP, SHH and SMO protein expression levels, whilst GFAP protein expression levels were significantly decreased compared with those in the DMSO control group (Fig. 6B). However, these Clo-induced changes were attenuated by treatment with CYC, 

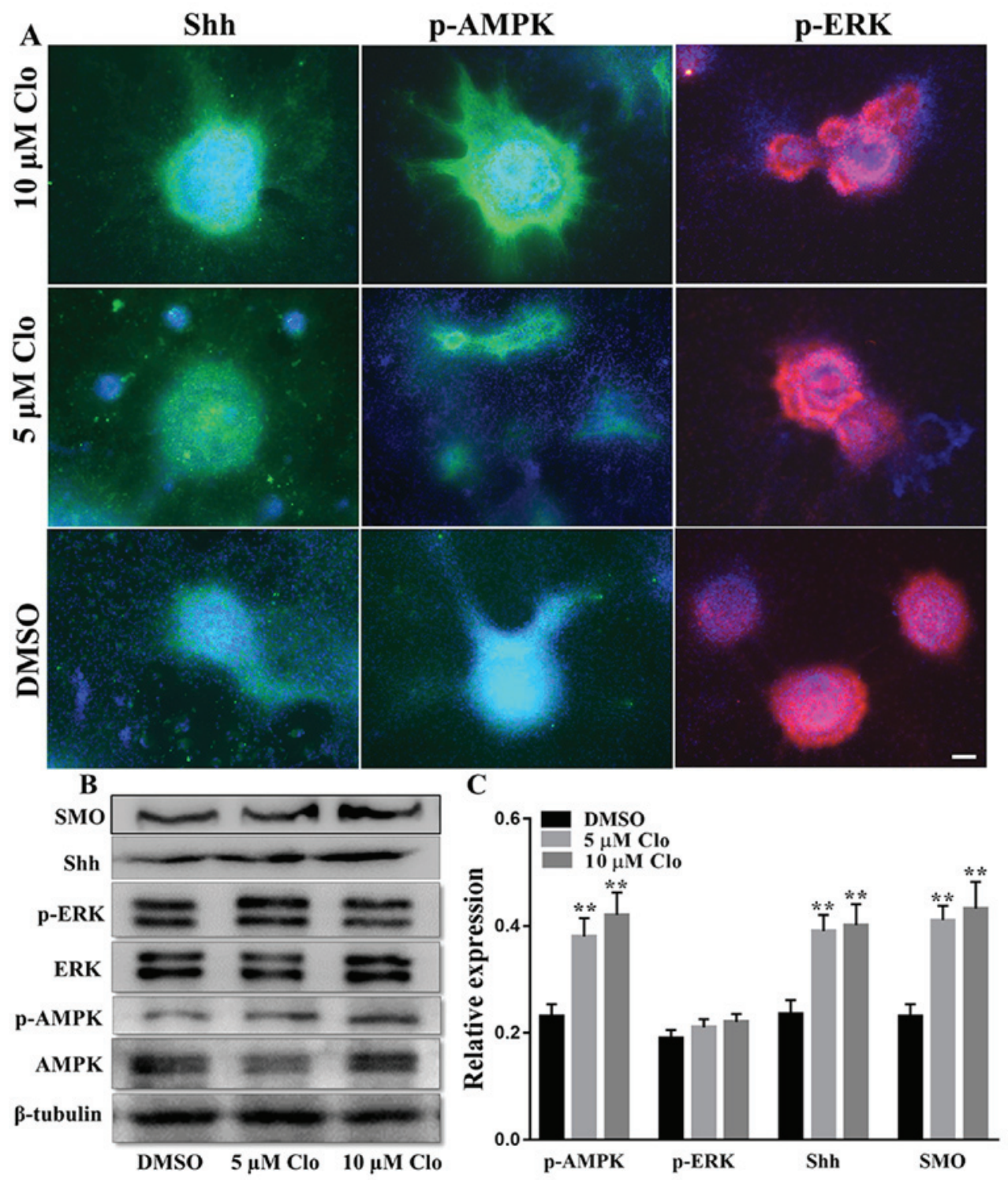

Figure 5. Effect of Clo on SHH, AMPK and ERK signaling pathways in NSCs. (A) Immunostaining of SHH, p-AMPK or p-ERK with DAPI nuclear staining in differentiated NSCs following treatment with Clo (5 or $10 \mu \mathrm{M}$ ) or DMSO (control). Scale bar $=25 \mu \mathrm{m}$. (B) Representative western blots of SMO, SHH, p-ERK, ERK, p-AMPK and AMPK in differentiated NSCs following treatment with Clo (5 or $10 \mu \mathrm{M}$ ) or DMSO (control). (C) Quantification of SMO, SHH, p-ERK/ERK and pAMPK/AMPK protein levels. Data are presented as the mean \pm standard error of the mean. $\mathrm{n}=5$. ${ }^{*} \mathrm{P}<0.05$ and ${ }^{* * *} \mathrm{P}<0.01 \mathrm{vs}$. DMSO. Clo, clobetasol propionate; SMO, smoothened homolog; SHH, sonic hedgehog; AMPK, AMP-activated protein kinase; ERK, mitogen-activated protein kinase 1; p, phosphorylated; NSCs, neural stem cells.
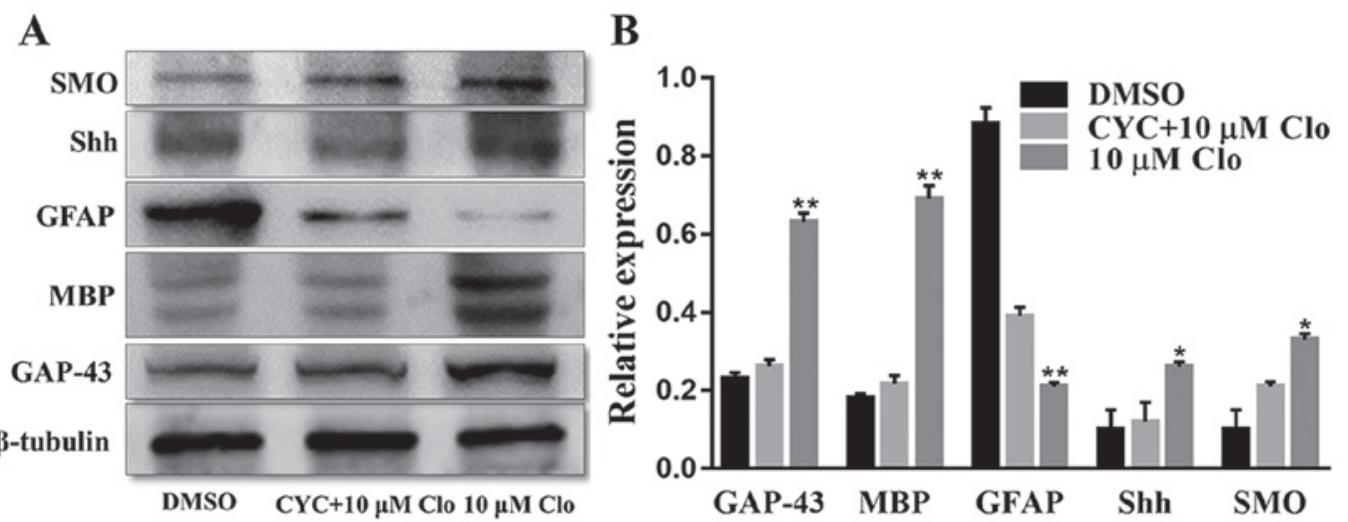

Figure 6. CYC inhibits Clo-induced NSC differentiation. (A) Representative western blots of GAP-43, MBP, GFAP, SHH and SMO in differentiated NSCs following treatment with $10 \mu \mathrm{M}$ Clo and $10 \mu \mathrm{M} \mathrm{CYC}$, or $10 \mu \mathrm{M}$ Clo alone. (B) Quantification of GAP-43, MBP, GFAP, SHH and SMO protein expression. Data are presented as the mean \pm standard error of the mean. $n=5$. ${ }^{*}<0.05$ and ${ }^{* *} \mathrm{P}<0.01$ vs. DMSO. CYC, cyclopamine; Clo, clobetasol propionate; NSC, neural stem cell; GAP-43, growth associated protein 43; MBP, myelin basic protein; GFAP, glial fibrillary acidic protein; SHH, sonic hedgehog; SMO, smoothened homolog. 

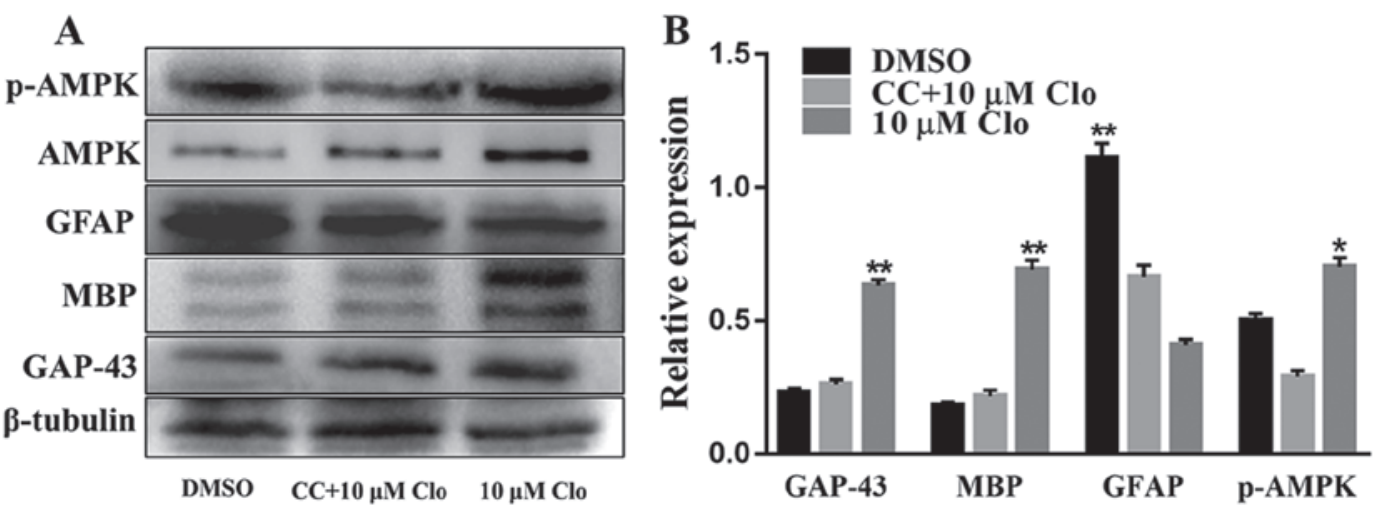

Figure 7. CC inhibits Clo-induced NSC differentiation. (A) Representative western blots of GAP-43, MBP, GFAP, AMPK and p-AMPK in differentiated NSCs following treatment with $10 \mu \mathrm{M}$ Clo and $5 \mu \mathrm{M}$ CC. (B) Quantification of GAP-43, MBP, GFAP, AMPK and p-AMPK proteins. Data are presented as the mean \pm standard error of the mean. $n=5$. ${ }^{*} \mathrm{P}<0.05$ and ${ }^{* *} \mathrm{P}<0.01$ vs. DMSO. CC, Compound $\mathrm{C}$; Clo, clobetasol propionate; NSC, neural stem cell; GAP-43, growth associated protein 43; MBP, myelin basic protein; GFAP, glial fibrillary acidic protein; AMPK, AMP-activated protein kinase; p, phosphorylated.
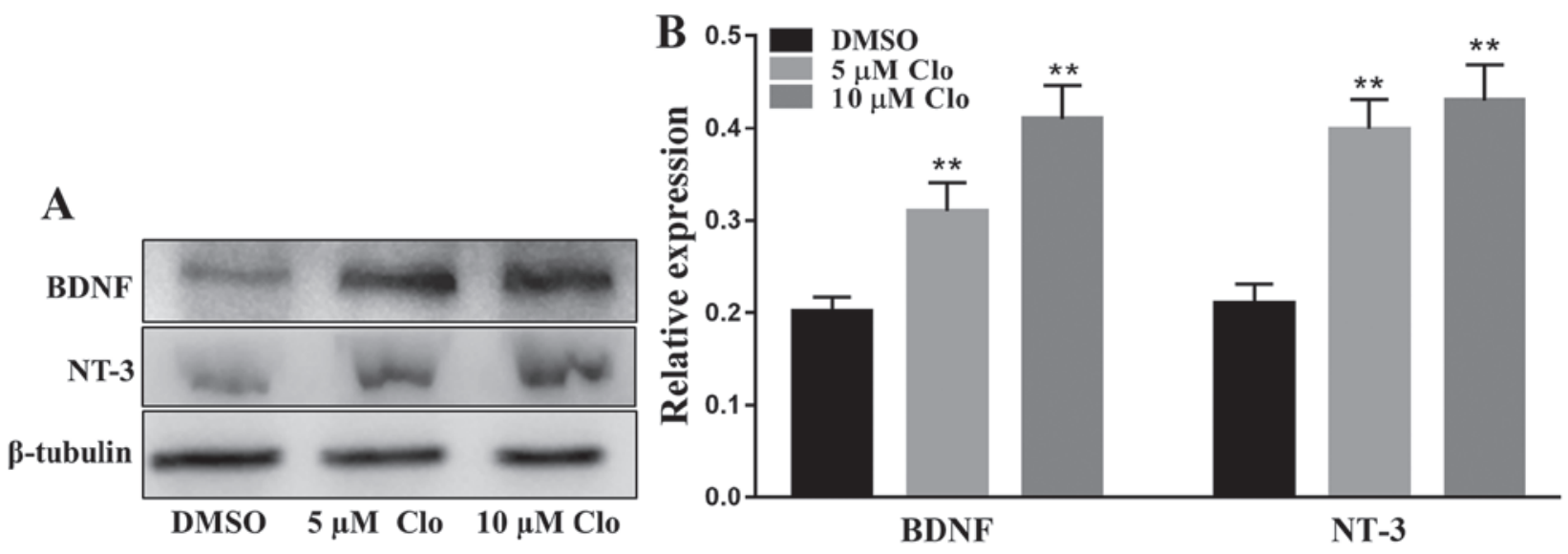

Figure 8. Clo increases the expression of brain-derived neurotrophic factors in NSCs. (A) Representative western blots of BDNF and NT-3 in differentiated NSCs following treatment with Clo $(5$ or $10 \mu \mathrm{M})$ or DMSO (control). (B) Quantification of BDNF and NT-3 proteins. Data are presented as the mean \pm standard error of the mean. $\mathrm{n}=5$. ${ }^{*} \mathrm{P}<0.05$ and ${ }^{* *} \mathrm{P}<0.01$ vs. DMSO. Clo, clobetasol propionate; NSC, neural stem cell; BDNF, brain-derived neurotrophic factor; NT-3, neurotrophin-3.

with no significant difference from the DMSO control group being detected (Fig. 6B).

Furthermore, the protein expression levels of GAP-43, MBP, GFAP, AMPK and p-AMPK were determined by western blot analysis in differentiated NSCs following treatment with $10 \mu \mathrm{M}$ Clo and $5 \mu \mathrm{M} \mathrm{CC}$ (Fig. 7A). Treatment with Clo alone significantly increased GAP-43, MBP and p-AMPK protein expression levels, whilst GFAP protein expression levels were significantly decreased compared with those in the DMSO control group (Fig. 7B). However, these Clo-induced changes were attenuated by treatment with $\mathrm{CC}$, with no significant differences detected compared with the DMSO control group (Fig. 7B).

Effect of Clo on brain-derived neurotrophic proteins. The protein expression levels of the neurotrophic factors NT-3 and BDNF were determined by western blot analysis in differentiated NSCs following treatment with Clo $(5$ or $10 \mu \mathrm{M})$ or DMSO (Fig. 8A). Treatment with Clo significantly increased NT-3 and BDNF protein expression levels compared with those in the DMSO control group (Fig. 8B).

\section{Discussion}

The present study demonstrated that Clo significantly enhanced NSC viability, and promotes the differentiation of NSCs into neurons and oligodendrocytes whilst inhibiting astrocyte differentiation. Furthermore, the present study indicated that Clo may promote NSC differentiation through the SHH and AMPK signaling pathways, providing a potentially novel mechanism underlying the therapeutic effect of Clo in CNS injury.

Previous studies demonstrated that Clo enhances differentiation in OPC cultures (17) and reduces myelin loss (22). However, whether Clo also affects NSC differentiation was unknown. Therefore, the aim of the present study was to investigate the effect of Clo on the differentiation of NSCs in vitro. NSCs were isolated from mouse embryonic brain tissue and expanded in vitro. It was observed that the NSCs, cultured as neurospheres, expressed nestin, a widely used marker of NSCs (23). Neurosphere formation can reflect the self-renewal capacity of cells (4). The MTT assay in the present study revealed that treatment with Clo significantly increased 
NSC viability at low concentrations $(2.5,5,7.5$ and $10 \mu \mathrm{M})$, consistent with a previous study (18). However, cell death was observed following treatment with Clo at concentrations $\geq 12.5 \mu \mathrm{M}$, indicating that high concentrations Clo may be toxic to NSCs. In addition, a recent study revealed that Clo activates the SHH signaling pathway, and as high concentrations of SHH inhibit cellular proliferation (24), high concentrations of Clo may inhibit NSC viability in a similar manner.

A previous study identified four glucocorticoids (halcinonide, fluticasone propionate, Clo and fluocinonide) as SMO agonists via a high-throughput screen of cells containing an SMO and $\beta$-arrestin-GFP reporter that binds to activated SMO (18). SMO is a component of the SHH signaling pathway (25), which can promote the expression of $\mathrm{HH}$ target genes by the Gli family of transcription factors (26). SHH is a morphogen produced by the notochord and the ventral floor plate that induces the development of various populations of ventral neurons and oligodendrocytes, and the proliferation of embryonic spinal cord OPCs and NSCs (27). SHH also serves a role in the development and maintenance of the nervous system (28). The current study demonstrated that treatment with Clo upregulates the expression of SMO and SHH proteins in differentiating NSCs. Although the molecular mechanism of Clo remains unknown, it can be hypothesized that Clo may activate the SHH signaling pathway by targeting SMO. Najm et al (17) reported that the glucocorticoid receptor may be involved in the process that regulates NSC differentiation into oligodendrocytes; however, the underlying molecular mechanism requires further investigation.

A previous study has demonstrated that Clo acts as a SMO agonist to promote myelin gene expression (18). Therefore, the Clo-associated enhancement of NSC viability and differentiation observed in the present study may be due to Clo having SHH-like and SMO agonist-like effects. Given that Clo is able to pass through the blood-brain barrier (16), Clo may exert a direct effect on the CNS. To further investigate whether Clo promotes the differentiation of NSCs through SHH signaling, SHH signaling was blocked in differentiating NSCs using CYC (29). Blocking the SHH signaling pathway exhibited a marked effect on Clo-induced NSC differentiation. Inhibition experiments further confirmed that Clo enhances NSC differentiation through the SHH signaling pathway.

Furthermore, the present study indicated that the AMPK signaling pathway may be involved in NSC proliferation and differentiation, as it was significantly upregulated following treatment with Clo. It is thought that AMPK maintains cellular energy homeostasis through the regulation of glucose and lipid metabolism (30). Although AMPK activation may inhibit cell proliferation $(31,32)$, the current study revealed that treatment with Clo enhances NSC viability. AMPK was previously demonstrated to be expressed in nerve cells (33). In the current study, p-AMPK was detected at elevated levels in differentiated NSCs. Inhibition experiments confirmed that the involvement of the AMPK signaling pathway in the regulation of NSC differentiation. A previous study reported that the administration of glucocorticoids may cause hyperphagia via the AMPK-neuropeptide Y (NPY) signaling pathway (34). NPY is involved in the modulation of the dynamics of NSC niches in the dentate gyrus and subventricular zone. Therefore, it may be hypothesized that AMPK-NPY is a target for Clo in NSCs. SHH signaling promotes polyamine biosynthesis in cerebellar granule cell precursors and this process is governed by AMPK (35). Although the mechanism underlying the activation of AMPK signaling by Clo was not elucidated in the current study, it is possible that SHH may be involved. Furthermore, it was previously revealed that Clo is able to upregulate the neural cell expression of NT-3 and BDNF, to promote neural differentiation and proliferation (36). Hence, Clo may enhance NSC differentiation and proliferation via the upregulation of neurotrophic factors.

The present study demonstrated that a high concentration $(10 \mu \mathrm{M})$ of Clo significantly suppressed the differentiation of NSCs into astrocytes. This was likely caused by the ability of corticosteroids to inhibit the inflammatory response in cells (37). Neuroinflammation is a feature associated with CNS disorders, including spinal cord injury and neurodegeneration, in which astrocytes serve critical roles (38). The reduced astrocyte differentiation capacity of Clo-treated NSCs may be a potential benefit towards the reduction of astrogliosis, the main cause of glial-scar formation, which limits axonal outgrowth and regeneration.

The current study has several limitations. In order to validate the results obtained in the current study, relevant in vivo studies and further mechanistic studies are required. In addition, human-derived stem cells should be used to ensure there are no species-specific differences.

In conclusion, the current study identified a novel, straightforward and efficient method to obtain differentiated cells with the typical morphological characteristics associated with NSC-derived neurons and oligodendrocytes. Furthermore, the well-known pharmacokinetic and pharmacodynamic properties of Clo combined with the results of the present study support the potential therapeutic application of Clo in human regenerative medicine.

\section{Acknowledgements}

Not applicable.

\section{Funding}

The present study was supported by grants from the National Natural Science Foundation of China (grant no. 81571830), Jiangsu Provincial Development Fund Project: Clinical Application of 3D Printing Technology in Complex Pelvic Fractures (grant no. YKK16228), Clinical Access Development Fund of Jiangsu University School of Medicine (grant nos. JLY20160185 and JLY20180040) and Social Development Project of Zhenjiang (grant no. 5561280012) from the Program for Scientific Research Innovation Team in Colleges and Universities of Jiangsu Province.

\section{Availability of data and materials}

The datasets used and/or analyzed during the present study are available from the corresponding author on reasonable request.

\section{Authors' contributions}

WS and ZZ designed the study. SB, YD and KY performed the experiments. WS and YZ prepared the manuscript. ZZ 
reviewed and edited the manuscript. All authors read and approved the final manuscript.

\section{Ethics approval and consent to participate}

Not applicable.

\section{Patient consent for publication}

Not applicable.

\section{Competing interests}

The authors declare that they have no competing interests.

\section{References}

1. Feder G, Murgai R and Quizon JB: Degeneration \& regeneration of the nervous system. Oxford University Press, 1928.

2. Vismara I, Papa S, Rossi F, Forloni G and Veglianese P: Current options for cell therapy in spinal cord injury. Trends Mol Med 23: 831-849, 2017.

3. Johansson CB, Momma S, Clarke DL, Risling M, Lendahl U and Frisén J: Identification of a neural stem cell in the adult mammalian central nervous system. Cell 96: 25-34, 1999.

4. Sundberg M, Savola S, Hienola A, Korhonen L and Lindholm D: Glucocorticoid hormones decrease proliferation of embryonic neural stem cells through ubiquitin-mediated degradation of cyclin D1. J Neurosci 26: 5402-5410, 2006.

5. Pathipati P, Gorba T, Scheepens A, Goffin V, Sun Y and Fraser M: Growth hormone and prolactin regulate human neural stem cell regenerative activity. Neuroscience 190: 409-427, 2011.

6. Koutmani Y and Karalis KP: Neural stem cells respond to stress hormones: Distinguishing beneficial from detrimental stress. Front Physiol 6: 77, 2015.

7. Ghareghani M, Sadeghi H, Zibara K, Danaei N, Azari H and Ghanbari A: Melatonin increases oligodendrocyte differentiation in cultured neural stem cells. Cell Mol Neurobiol 37: 1319-1324, 2017.

8. Nakamura M and Toyama Y: Transplantation of neural stem cells into spinal cord after injury. Nihon Rinsho 61: 463-468, 2003 (In Japanese).

9. Corsini NS, Sancho-Martinez I, Laudenklos S, Glagow D, Kumar S, Letellier E, Koch P, Teodorczyk M, Kleber S, Klussmann S, et al: The death receptor CD95 activates adult neural stem cells for working memory formation and brain repair. Cell Stem Cell 5: 178-190, 2009.

10. Patel S, Saha A, Buntz S, Kurtzberg J and Balber A: Neuronal and glial cell composition in a mouse brain slice culture model is useful in developing human cord blood derived cellular therapies for neonatal hypoxic-ischemic brain injury. Cytotherapy 16 (Suppl): S61, 2014

11. Fawcett JW and Asher RA: The glial scar and central nervous system repair. Brain Res Bull 49: 377-391, 1999.

12. Au WL, Skinner MF, Benfeldt E, Verbeeck R-K and Kanfer I: Application of dermal microdialysis for the determination of bioavailability of clobetasol propionate applied to the skin of human subjects. Skin Pharmacol Physiol 25: 17-24, 2012.

13. Olsen JA and Akirav EM: Remyelination in multiple sclerosis: cellular mechanisms and novel therapeutic approaches. J Neurosci Res 93: 687-696, 2015.

14. Bove RM and Green AJ: Remyelinating pharmacotherapies in multiple sclerosis. Neurotherapeutics 14: 894-904, 2017.

15. Cole KLH, Early JJ and Lyons DA: Drug discovery for remyelination and treatment of MS. Glia 65: 1565-1589, 2017.

16. Yao X, Su T and Verkman AS: Clobetasol promotes remyelination in a mouse model of neuromyelitis optica. Acta Neuropathol Commun 4: 42, 2016.

17. Najm FJ, Madhavan M, Zaremba A, Shick E, Karl RT, Factor DC, Miller TE, Nevin ZS, Kantor C, Sargent A, et al: Drug-based modulation of endogenous stem cells promotes functional remyelination in vivo. Nature 522: 216-220, 2015.
18. Wang J, Lu J, Bond MC, Chen M, Ren XR, Lyerly HK, Barak LS and Chen W: Identification of select glucocorticoids as Smoothened agonists: Potential utility for regenerative medicine. Proc Natl Acad Sci USA 107: 9323-9328, 2010.

19. Lu P, Graham L, Wang Y, Wu D and Tuszynski M: Promotion of survival and differentiation of neural stem cells with fibrin and growth factor cocktails after severe spinal cord injury. J V is Exp: e50641, 2014

20. Li J, Wang G, Wang C, Zhao Y, Zhang H, Tan Z, Song Z, Ding M and Deng H: MEK/ERK signaling contributes to the maintenance of human embryonic stem cell self-renewal. Differentiation 75: 299-307, 2010.

21. Zang Y, Yu LF, Nan FJ, Feng LY and Li J: AMP-activated protein kinase is involved in neural stem cell growth suppression and cell cycle arrest by 5 -aminoimidazole-4-carboxamide-1-beta-Dribofuranoside and glucose deprivation by down-regulating phospho-retinoblastoma protein and cyclin D. J Biol Chem 284: 6175-6184, 2009.

22. Hampton T: Drugs that treat skin conditions may help combat multiple sclerosis. JAMA 313: 2114, 2015.

23. Park D, Xiang AP, Mao FF, Zhang L, Di CG, Liu XM, Shao Y, Ma BF, Lee JH, Ha KS, et al: Nestin is required for the proper self-renewal of neural stem cells. Stem Cells 28: 2162-2171,2011.

24. Canettieri G, Coni S, Laura DM and Sdruscia G: Non-canonical Hedgehog/AMPK-mediated control of polyamine metabolism is required for medulloblastoma growth. Eur J Cancer 61 (Suppl): S72, 2016.

25. Ye W, Shimamura K, Rubenstein JLR, Hynes MA and Rosenthal A: FGF and SHH signals control dopaminergic and serotonergic cell fate in the anterior neural plate. Cell 93: 755-766, 1998.

26. Sinha S and Chen JK: Purmorphamine activates the Hedgehog pathway by targeting smoothened. Nat Chem Biol 2: 29-30, 2006.

27. Zhang Z, Li Z, Deng W, He Q, Wang Q, Shi W, Chen Q, Yang W, Spector M, Gong A, et al: Ectoderm mesenchymal stem cells promote differentiation and maturation of oligodendrocyte precursor cells. Biochem Biophys Res Commun 480: 727-733, 2016.

28. Scadden DT: The stem-cell niche as an entity of action. Nature 441: 1075-1079, 2006.

29. Chen JK, Taipale J, Cooper MK and Beachy PA: Inhibition of Hedgehog signaling by direct binding of cyclopamine to Smoothened. Genes Dev 16: 2743-2748, 2002.

30. Krieg S, Lüscher B, Vervoorts J and Dohmen M: Studying the role of AMPK in autophagy. Methods Mol Biol 1732: 373-391, 2018.

31. Garcia D and Shaw RJ: AMPK: Mechanisms of cellular energy sensing and restoration of metabolic balance. Mol Cell 66: 789-800, 2017.

32. Ronnett GV, Ramamurthy S, Kleman AM, Landree LE and Aja S: AMPK in the brain: Its roles in energy balance and neuroprotection. J Neurochem 109 (Suppl 1): S17-S23, 2009.

33. Rich B, Scadeng M, Yamaguchi M, Wagner PD and Breen EC: Skeletal myofiber VEGF is required for the exercise training-induced increase in dentate gyrus neuronal precursor cells. J Physiol 595: 5931-5943, 2017.

34. Liu L, Song Z, Jiao H and Lin H: Glucocorticoids increase NPY gene expression via hypothalamic AMPK signaling in broiler chicks. Endocrinology 155: 2190-2198, 2014.

35. Di Magno L, Basile A, Coni S, Manni S, Sdruscia G, D'Amico D, Antonucci L, Infante P, De Smaele E, Cucchi D, et al: The energy sensor AMPK regulates Hedgehog signaling in human cells through a unique Gli1 metabolic checkpoint. Oncotarget 7: 9538-9549, 2016.

36. Marsh SE and Blurton-Jones M: Neural stem cell therapy for neurodegenerative disorders: The role of neurotrophic support. Neurochem Int 106: 94-100, 2017.

37. Jauregui-Huerta F, Ruvalcaba-Delgadillo Y, Gonzalez-Perez O, Gonzalez-Castañeda R, Garcia-Estrada J and Luquin S: Responses of glial cells to stress and glucocorticoids. Curr Immunol Rev 6: 195-204, 2010

38. Blancosuárez E, Caldwell AL and Allen NJ: Role of astrocyte-synapse interactions in CNS disorders. J Physiol 595: 1903-1916, 2017.

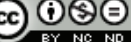

This work is licensed under a Creative Commons Attribution-NonCommercial-NoDerivatives 4.0 International (CC BY-NC-ND 4.0) License. 